\title{
A Version of Uncertainty Principle for Quaternion Linear Canonical Transform
}

\author{
Mawardi Bahri $\mathbb{D}^{1},{ }^{1}$ Resnawati, ${ }^{2}$ and Selvy Musdalifah ${ }^{2}$ \\ ${ }^{1}$ Department of Mathematics, Hasanuddin University, Makassar 90245, Indonesia \\ ${ }^{2}$ Department of Mathematics, Tadulako University, Palu 94148, Indonesia \\ Correspondence should be addressed to Mawardi Bahri; mawardibahri@gmail.com
}

Received 2 October 2017; Revised 18 March 2018; Accepted 2 April 2018; Published 7 May 2018

Academic Editor: Feyzi Başar

Copyright (C) 2018 Mawardi Bahri et al. This is an open access article distributed under the Creative Commons Attribution License, which permits unrestricted use, distribution, and reproduction in any medium, provided the original work is properly cited.

In recent years, the two-dimensional (2D) quaternion Fourier and quaternion linear canonical transforms have been the focus of many research papers. In the present paper, based on the relationship between the quaternion Fourier transform (QFT) and the quaternion linear canonical transform (QLCT), we derive a version of the uncertainty principle associated with the QLCT. We also discuss the generalization of the Hausdorff-Young inequality in the QLCT domain.

\section{Introduction}

The quaternion Fourier transform (QFT) is an extension of the classical two-dimensional Fourier transform (FT) [1-4] in the framework of quaternion algebra. It plays an important role in the representation of the two-dimensional quaternion signals. A number of useful properties of the extended transform are generalizations of the corresponding properties of the FT with some modifications (see, e.g., [515]). The QFT has found many applications in color image processing and signal analysis; we refer the reader to [1619] and the references mentioned therein. An extension of the QFT in the framework of the classical linear canonical transform (LCT) (see [20-22]), known as the quaternion linear canonical transform, has received much attention in recent years. Due to the several definitions of the QFT, there are basically three ways of obtaining the quaternion linear canonical transform (QLCT): the right-sided quaternion linear canonical transform, the left-sided quaternion linear canonical transform, and the two-sided quaternion linear canonical transform. The right-sided quaternion linear canonical transform is obtained by substituting the Fourier kernel with the right-sided QFT kernel in the LCT definition, and so on. Recent works related to some important properties of the QLCT such as Parseval's theorem, reconstruction formula, and component-wise uncertainty principles were also published [18, 23-25]. It was found that the properties of the QLCT are extensions of the corresponding version of the QFT with some modifications.

On the other hand, the uncertainty principle plays one important role in signal processing. It describes a function and its FT, which cannot both be simultaneously sharply localized. One example of this is the Heisenberg uncertainty principle concerning position and momentum wave functions in quantum physics. In signal processing, an uncertainty principle states that the product of the variances of the signal in the time and frequency domains has a lower bound. Up till now, several attempts have been made to extend the uncertainty principles associated with the QFT and QLCT domains. The component-wise and directional uncertainty principles associated with the QFT were proposed in [11]. In $[26,27]$, the authors established a component-wise uncertainty principle for the QLCT and proved that the equality is achieved for optimal quaternion Gaussian function. Recently, the authors [23] proposed the logarithmic uncertainty principle associated with the QLCT which is the generalization of the logarithmic uncertainty principle for the QFT.

Therefore, the main objective of the present paper is to establish full uncertainty principle for the two-sided QLCT, which is a new general form of component-wise uncertainty principle for the two-sided QLCT. This uncertainty principle is derived using the connection between the QFT and QLCT. 
We also obtain full uncertainty principle for the rightsided QLCT using a relation between the right-sided QLCT and two-sided QLCT. We also derive the Hausdorff-Young inequality associated with the two-sided QLCT.

This paper is organized as follows. In Section 2, we briefly review the basic knowledge of quaternion and its split used in the next section. In Section 3, we introduce the definition of the QFT and the QLCT. The relationship between the QFT and the QLCT is also discussed in this section. In Section 4, we derive a new version uncertainty principle associated with the QLCT, which shows that the spread of a quaternionvalued function and its QLCT are inversely proportional. Section 5 concludes this paper.

\section{Preliminaries}

In this section, let us briefly recall some basic definitions and properties of the quaternions (for more details, see [28]).

2.1. Quaternions. The quaternions, a generalization of complex numbers, are members of a noncommutative division algebra. The set of quaternions is denoted by $\mathbb{H}$. Every element

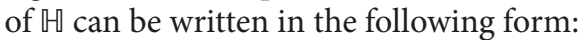

$$
\mathbb{H}=\left\{q=q_{0}+\mathbf{i} q_{1}+\mathbf{j} q_{2}+\mathbf{k} q_{3} ; q_{0}, q_{1}, q_{2}, q_{3} \in \mathbb{R}\right\},
$$

with the units $\mathbf{i}, \mathbf{j}, \mathbf{k}$, which obey the following:

$$
\begin{aligned}
& \mathbf{i j}=-\mathbf{j i}=\mathbf{k}, \\
& \mathbf{j k}=-\mathbf{k} \mathbf{j}=\mathbf{i}, \\
& \mathbf{k i}=-\mathbf{i k}=\mathbf{j}, \\
& \mathbf{i}^{2}=\mathbf{j}^{2}=\mathbf{k}^{2}=\mathbf{i j k}=-1 .
\end{aligned}
$$

For a quaternion $q=q_{0}+\mathbf{i} q_{1}+\mathbf{j} q_{2}+\mathbf{k} q_{3} \in \mathbb{H}, q_{0}$ is simply called the scalar part of $q$ denoted by $\operatorname{Sc}(q)$ and $\mathbf{q}=\mathbf{i} q_{1}+\mathbf{j} q_{2}+\mathbf{k} q_{3}$ is called the vector part of $q$ denoted by $\operatorname{Vec}(q)$.

Let $p, q \in \mathbb{H}$ and $\mathbf{p}, \mathbf{q}$ be their vector parts, respectively. Equation (2) yields the quaternionic multiplication $q p$ as

$$
q p=q_{0} p_{0}+\mathbf{q} \cdot \mathbf{p}+q_{0} \mathbf{p}+p_{0} \mathbf{q}+\mathbf{q} \times \mathbf{p}
$$

where

$$
\begin{aligned}
\mathbf{q} \cdot \mathbf{p}= & -\left(q_{1} p_{1}+q_{2} p_{2}+q_{3} p_{3}\right), \\
\mathbf{q} \times \mathbf{p}= & \mathbf{i}\left(q_{2} p_{3}-q_{3} p_{2}\right)+\mathbf{j}\left(q_{3} p_{1}-q_{1} p_{3}\right) \\
& +\mathbf{k}\left(q_{1} p_{2}-q_{2} p_{1}\right) .
\end{aligned}
$$

Analogously to the complex case, a quaternionic conjugation $\bar{q}$ is given by

$$
\bar{q}=q_{0}-\mathbf{i} q_{1}-\mathbf{j} q_{2}-\mathbf{k} q_{3}
$$

which leads to the anti-involution; that is,

$$
\overline{q p}=\bar{p} \bar{q} .
$$

With the help of (5), we get the norm or modulus of $q \in \mathbb{H}$ as

$$
|q|=\sqrt{q \bar{q}}=\sqrt{q_{0}^{2}+q_{1}^{2}+q_{2}^{2}+q_{3}^{2}} .
$$

One can easily verify that

$$
\begin{aligned}
&|q p|=|q||p|, \\
&|q+p| \leq|q|+|p|, \\
& \forall p, q \in \mathbb{U} .
\end{aligned}
$$

Using conjugate (5) and the modulus of $q$, we can define the inverse of $q \in \mathbb{U} \backslash\{0\}$ as

$$
q^{-1}=\frac{\bar{q}}{|q|^{2}}
$$

which shows that $\mathbb{H}$ is a normed division algebra.

In quaternionic notation, we may define an inner product for quaternion-valued functions $f, g: \mathbb{R}^{2} \rightarrow \mathbb{H}$ as follows:

$$
(f, g)=\int_{\mathbb{R}^{2}} f(\mathbf{x}) \overline{g(\mathbf{x})} d \mathbf{x}, \quad d \mathbf{x}=d x_{1} d x_{2}
$$

with symmetric real scalar part

$$
\begin{aligned}
\langle f, g\rangle & =(f, g)_{0}=\frac{1}{2}[(f, g)+(g, f)] \\
& =\int_{\mathbb{R}^{2}} \operatorname{Sc}(f(\mathbf{x}) \bar{g}(\mathbf{x})) d \mathbf{x} .
\end{aligned}
$$

In particular, for $f=g$, we obtain the $L^{2}\left(\mathbb{R}^{2} ; \mathfrak{U}\right)$-norm

$$
\|f\|=\sqrt{\langle f, f\rangle}=\left(\int_{\mathbb{R}^{2}}|f(\mathbf{x})|^{2} d \mathbf{x}\right)^{1 / 2} .
$$

A quaternion module $L^{2}\left(\mathbb{R}^{2} ; \mathbb{H}\right)$ is then defined as

$$
L^{2}\left(\mathbb{R}^{2} ; \mathbb{H}\right)=\left\{f \mid f: \mathbb{R}^{2} \longrightarrow \mathbb{H},\|f\|<\infty\right\} .
$$

2.2. Split Quaternion and Properties. In this section, we study some of the basic formulas of split quaternion (see [9]), which will be used to prove the fundamental results in the sequel.

Definition 1. For two quaternion square roots $\mu, v$ such that $\mu^{2}=\nu^{2}=-1$, one may express a quaternion $q$ as

$$
q=q_{-}+q_{+}, \quad q_{ \pm}=\frac{1}{2}(q \pm \mu q \nu) .
$$

For the special case of $\mu=\nu$, any quaternion $q$ may be split up into commuting and anticommuting parts with respect to $\mu$; that is,

$$
\begin{aligned}
& \mu q_{-}=q_{-} \mu \\
& \mu q_{+}=-q_{+} \mu .
\end{aligned}
$$

It easily seems that the commuting and anticommuting parts satisfy the interesting properties:

$$
\begin{aligned}
\mu^{2} & =\mu_{-}^{2}+\mu_{+}^{2}=-1 \\
\mu_{+} \mu_{-}+\mu_{-} \mu_{+} & =0 .
\end{aligned}
$$


We learn from the above equation that

$$
q_{ \pm} e^{\mu \theta}=e^{\mp \mu \theta} q_{ \pm}
$$

where

$$
\begin{aligned}
& \cos \theta=\frac{q_{0}}{|q|}, \\
& \sin \theta=\frac{\sqrt{q_{1}^{2}+q_{2}^{2}+q_{3}^{2}}}{|q|} .
\end{aligned}
$$

In particular, taking $\mu=\mathbf{i}$ and $\nu=\mathbf{j}$, (14) becomes

$$
q=q_{+}+q_{-}, \quad q_{ \pm}=\frac{1}{2}(q \pm \mathbf{i} q \mathbf{j})
$$

The above gives

$$
\begin{aligned}
q_{ \pm} & =\left\{\left(q_{0} \pm q_{3}\right)+\mathbf{i}\left(q_{1} \mp q_{2}\right)\right\} \frac{1 \pm \mathbf{k}}{2} \\
& =\frac{1 \pm \mathbf{k}}{2}\left\{\left(q_{0} \pm q_{3}\right)+\mathbf{j}\left(q_{2} \mp q_{1}\right)\right\} .
\end{aligned}
$$

This leads to the following modulus identity:

$$
|q|^{2}=\left|q_{-}\right|^{2}+\left|q_{+}\right|^{2}
$$

Furthermore, one can obtain

$$
\operatorname{Sc}\left(p_{+} \bar{q}_{-}\right)=0 .
$$

\section{Relationship between Quaternion Fourier Transform (QFT) and Quaternion Linear Canonical Transform (QLCT)}

In this section, we introduce the QFT and its relationship to the QLCT. We begin by introducing different types of the QFT.

Definition 2 (two-sided, right-sided, and left-sided QFTs). The two-sided, right-sided, and left-sided quaternion Fourier transforms (QFTs) of $f \in L^{1}\left(\mathbb{R}^{2} ; \mathbb{H}\right)$ are given by, respectively,

$$
\begin{aligned}
& \mathscr{F}_{q}\{f\}(\boldsymbol{\omega})=\int_{\mathbb{R}^{2}} e^{-\mathbf{i} \omega_{1} x_{1}} f(\mathbf{x}) e^{-\mathbf{j} \omega_{2} x_{2}} d \mathbf{x} \\
& \mathscr{F}_{q}^{r}\{f\}(\boldsymbol{\omega})=\int_{\mathbb{R}^{2}} f(\mathbf{x}) e^{-\mathbf{i} \omega_{1} x_{1}} e^{-\mathbf{j} \omega_{2} x_{2}} d \mathbf{x} \\
& \mathscr{F}_{q}^{l}\{f\}(\boldsymbol{\omega})=\int_{\mathbb{R}^{2}} e^{-\mathbf{i} \omega_{1} x_{1}} e^{-\mathbf{j} \omega_{2} x_{2}} f(\mathbf{x}) d \mathbf{x},
\end{aligned}
$$

where $\mathbf{x}=x_{1} \mathbf{e}_{1}+x_{2} \mathbf{e}_{2}, \boldsymbol{\omega}=\omega_{1} \mathbf{e}_{1}+\omega_{2} \mathbf{e}_{2}$, and the quaternion exponential product $e^{-\mathbf{i} \omega_{1} x_{1}} e^{-\mathbf{j} \omega_{2} x_{2}}$ is the quaternion Fourier kernel. From (24), we get the partial right-sided QFT:

$$
\begin{aligned}
& \mathscr{F}_{q}^{r, \mathbf{i}}\{f\}\left(\omega_{1}, x_{2}\right)=\int_{\mathbb{R}^{2}} f(\mathbf{x}) e^{-\mathbf{i} \omega_{1} x_{1}} d x_{1} \\
& \mathscr{F}_{q}^{r, \mathbf{j}}\{f\}\left(x_{1}, \omega_{2}\right)=\int_{\mathbb{R}^{2}} f(\mathbf{x}) e^{-\mathbf{j} \omega_{2} x_{2}} d x_{2} .
\end{aligned}
$$

It is not difficult to check that the relationship between the two-sided QFT and the right-sided QFT takes the form

$$
\begin{aligned}
\mathscr{F}_{q}\{f\}(\boldsymbol{\omega})= & \mathscr{F}_{q}^{r}\left\{f_{0}+\mathbf{i} f_{1}\right\}(\boldsymbol{\omega}) \\
& +\mathscr{F}_{q}^{r}\left\{\mathbf{j} f_{2}+\mathbf{k} f_{3}\right\}\left(-\omega_{1}, \omega_{2}\right) .
\end{aligned}
$$

Definition 3 (left-sided, right-sided, and two-sided QLCTs). Suppose that $A_{1}=\left(a_{1}, b_{1}, c_{1}, d_{1}\right)$ and $A_{2}=\left(a_{2}, b_{2}, c_{2}, d_{2}\right)$ are real matrix parameters satisfying $\operatorname{det}\left(A_{1}\right)=\operatorname{det}\left(A_{2}\right)=1$. The left-sided, right-sided, and two-sided QLCTs of a quaternion signal $f \in L^{1}\left(\mathbb{R}^{2} ; \mathbb{H}\right)$ are defined by, respectively,

$$
\begin{aligned}
& L_{A_{1}, A_{2}}^{(l), \mathbb{Q}}\{f\}(\boldsymbol{\omega}) \\
& =\int_{\mathbb{R}^{2}} K_{A_{1}}\left(x_{1}, \omega_{1}\right) K_{A_{2}}\left(x_{2}, \omega_{2}\right) f(\mathbf{x}) d \mathbf{x} \\
& L_{A_{1}, A_{2}}^{(r) \mathbb{H}}\{f\}(\boldsymbol{\omega}) \\
& =\int_{\mathbb{R}^{2}} f(\mathbf{x}) K_{A_{1}}\left(x_{1}, \omega_{1}\right) K_{A_{2}}\left(x_{2}, \omega_{2}\right) d \mathbf{x} \\
& L_{A_{1}, A_{2}}^{\mathbb{Q}}\{f\}(\boldsymbol{\omega}) \\
& =\int_{\mathbb{R}^{2}} K_{A_{1}}\left(x_{1}, \omega_{1}\right) f(\mathbf{x}) K_{A_{2}}\left(x_{2}, \omega_{2}\right) d \mathbf{x},
\end{aligned}
$$

where the kernel functions of the QLCT above are given by

$$
\begin{aligned}
& K_{A_{1}}\left(x_{1}, \omega_{1}\right) \\
& = \begin{cases}\frac{1}{\sqrt{2 \pi b_{1}}} e^{(\mathbf{i} / 2)\left(\left(a_{1} / b_{1}\right) x_{1}^{2}-\left(2 / b_{1}\right) x_{1} \omega_{1}+\left(d_{1} / b_{1}\right) \omega_{1}^{2}-\pi / 2\right)}, & \text { for } b_{1} \neq 0 \\
\sqrt{d_{1}} e^{\mathbf{i}\left(c_{1} d_{1} / 2\right) \omega_{1}^{2}}, & \text { for } b_{1}=0,\end{cases} \\
& K_{A_{2}}\left(x_{2}, \omega_{2}\right) \\
& = \begin{cases}\frac{1}{\sqrt{2 \pi b_{2}}} e^{(\mathbf{j} / 2)\left(\left(a_{2} / b_{2}\right) x_{2}^{2}-\left(2 / b_{2}\right) x_{2} \omega_{2}+\left(d_{2} / b_{2}\right) \omega_{2}^{2}-\pi / 2\right)}, & \text { for } b_{2} \neq 0 \\
\sqrt{d_{2}} e^{\mathbf{j}\left(c_{2} d_{2} / 2\right) \omega_{2}^{2}}, & \text { for } b_{2}=0 .\end{cases}
\end{aligned}
$$

From the definition of the QLCT, we can easily see that when $b_{1} b_{2}=0$ and $b_{1}=b_{2}=0$, the QLCT of a signal is essentially a quaternion chirp multiplication. Therefore, in this work, we always assume $b_{1} b_{2} \neq 0$. As a special case, when $A_{1}=A_{2}=\left(a_{i}, b_{i}, c_{i}, d_{i}\right)=(0,1,-1,0)$, for $i=1,2$, the QLCT definition (30) will lead to the QFT definition; that is,

$$
\begin{aligned}
& L_{A_{1}, A_{2}}^{\mathbb{\boxplus}}\{f\}(\boldsymbol{\omega}) \\
& \quad=\int_{\mathbb{R}^{2}} \frac{e^{-\mathbf{i}(\pi / 4)}}{\sqrt{2 \pi}} e^{-\mathbf{i} \omega_{1} x_{1}} f(\mathbf{x}) e^{-\mathbf{j} \omega_{2} x_{2}} \frac{e^{-\mathbf{j}(\pi / 4)}}{\sqrt{2 \pi}} d \mathbf{x} \\
& \quad=\frac{e^{-\mathbf{i}(\pi / 4)}}{\sqrt{2 \pi}} \mathscr{F}_{q}\{f\}(\boldsymbol{\omega}) \frac{e^{-\mathbf{j}(\pi / 4)}}{\sqrt{2 \pi}} .
\end{aligned}
$$

The following lemma describes the general relationship between the two-sided QFT and the two-sided QLCT of 2D quaternion-valued signals. 
Lemma 4. The QLCT of a signal $f$ with matrix parameters $A_{1}=\left(a_{1}, b_{1}, c_{1}, d_{1}\right)$ and $A_{2}=\left(a_{2}, b_{2}, c_{2}, d_{2}\right)$ can be seen as the QFT of the signal $f$ in the following form:

$$
\begin{aligned}
& L_{A_{1}, A_{2}}^{\mathbf{H}}\{f\}(\boldsymbol{\omega})=\frac{e^{-\mathbf{i}(\pi / 4)}}{\sqrt{2 \pi b_{1}}} \\
& \cdot e^{\left(\mathbf{i d} d_{1} / 2 b_{1}\right) \omega_{1}^{2}} \mathscr{F}_{q}\left\{e^{\mathbf{i}\left(a_{1} / 2 b_{1}\right) x_{1}^{2}} f(\mathbf{x}) e^{\mathbf{j}\left(a_{2} / 2 b_{2}\right) x_{2}^{2}}\right\} \\
& \cdot\left(\frac{\omega_{1}}{b_{1}}, \frac{\omega_{2}}{b_{2}}\right) \frac{e^{-\mathbf{j}(\pi / 4)}}{\sqrt{2 \pi b_{2}}} e^{\left(\mathbf{j} d_{2} / 2 b_{2}\right) \omega_{2}^{2}} .
\end{aligned}
$$

We introduce

$$
h(\mathbf{x})=e^{\mathbf{i}\left(a_{1} / 2 b_{1}\right) x_{1}^{2}} \frac{e^{-\mathbf{i}(\pi / 4)}}{\sqrt{2 \pi b_{1}}} f(\mathbf{x}) \frac{e^{-\mathbf{j}(\pi / 4)}}{\sqrt{2 \pi b_{2}}} e^{\mathbf{j}\left(a_{2} / 2 b_{2}\right) x_{2}^{2}} .
$$

This implies that (33) can be rewritten in the form

$$
\begin{aligned}
\mathscr{F}_{q} & \{h\}\left(\frac{\omega_{1}}{b_{1}}, \frac{\omega_{2}}{b_{2}}\right) \\
= & e^{-\left(\mathbf{i} d_{1} / 2 b_{1}\right) \omega_{1}^{2}} L_{A_{1}, A_{2}}^{\mathbf{H}}\{h\}(\boldsymbol{\omega}) e^{-\left(\mathbf{j} d_{2} / 2 b_{2}\right) \omega_{2}^{2}} .
\end{aligned}
$$

Further, we have the following lemma which describes a relation between the right-sided QLCT and the two-sided QLCT of 2D quaternion-valued signals.

Lemma 5. For $f \in L^{1}\left(\mathbb{R}^{2} ; \mathbb{H}\right)$, one has

$$
\begin{aligned}
L_{A_{1}, A_{2}}^{(r), \mathbf{H}}\{f\}(\boldsymbol{\omega})= & L_{A_{1}, A_{2}}^{\mathbf{H}}\left\{f_{0}+\mathbf{i} f_{1}\right\}(\boldsymbol{\omega}) \\
& +L_{A_{1}^{*}, A_{2}^{*}}^{\mathbf{H}}\left\{\mathbf{j} f_{2}+\mathbf{k} f_{3}\right\}\left(-\omega_{1}, \omega_{2}\right), \\
L_{A_{1}, A_{2}}^{\mathbf{H}}\{f\}(\boldsymbol{\omega})= & L_{A_{1}, A_{2}}^{(r), \mathbf{H}}\left\{f_{0}+\mathbf{i} f_{1}\right\}(\boldsymbol{\omega}) \\
& +L_{A_{1}^{*}, A_{2}^{*}}^{(r), \mathbf{H}}\left\{\mathbf{j} f_{2}+\mathbf{k} f_{3}\right\}\left(-\omega_{1}, \omega_{2}\right),
\end{aligned}
$$

where the matrix parameters $A_{1}^{*}=\left(a_{1},-b_{1}, c_{1}, d_{1}\right)$ and $A_{2}^{*}=$ $\left(a_{2},-b_{2}, c_{2}, d_{2}\right)$.

The following lemma allows us to represent the rightsided QLCT to the single right-sided QFT of 2D quaternionvalued signals.

Lemma 6. For $f \in L^{1}\left(\mathbb{R}^{2} ; \mathfrak{H}\right)$, one has

$$
\begin{gathered}
L_{A_{1}, A_{2}}^{(r), \mathbf{H}}\{f\}(\boldsymbol{\omega})=\mathscr{F}_{q}^{r, \mathbf{j}}\left\{\mathscr{F}_{q}^{r, \mathbf{i}}\{g(\mathbf{x})\}\left(\frac{\omega_{1}}{b_{1}}, x_{2}\right)\right. \\
\left.\cdot e^{\mathbf{i}\left(d_{1} / 2 b_{1}\right) \omega_{1}^{2}} \frac{e^{\mathbf{j}\left(\left(a_{2} / 2 b_{2}\right) x_{2}^{2}-\pi / 4\right)}}{\sqrt{2 \pi b_{2}}}\right\}\left(\frac{\omega_{1}}{b_{1}}, \frac{\omega_{2}}{b_{2}}\right) .
\end{gathered}
$$

Proof. Proofs of Lemmas 4, 5, and 6 are straightforward and are therefore omitted for brevity.

\section{A Version of Uncertainty Principle Associated with QLCT}

The classical uncertainty principle of harmonic analysis describes that a nontrivial function and its Fourier transform cannot be sharply localized simultaneously. In quantum mechanics, the uncertainty principle asserts that one cannot at the same time be certain of the position and of the velocity of an electron (or any particle). Let us now establish a version of the uncertainty principle associated with the QLCT. However, before proceeding the statement of this main result, we need to introduce a modified uncertainty principle for the QFT as follows (see [29] for more details).

Theorem 7 (the two-sided QFT uncertainty principle). Let $\delta\left(\mathbb{R}^{2} ; \mathbb{M}\right)$ be the quaternion Schwartz space. If the quaternionvalued function $f \in S\left(\mathbb{R}^{2} ; \mathbb{M}\right)$, then the following inequality holds:

$$
\begin{aligned}
& \int_{\mathbb{R}^{2}} x_{1}^{2}|f(\mathbf{x})|^{2} x_{2}^{2} d \mathbf{x} \int_{\mathbb{R}^{2}} \omega_{1}^{2}\left|\mathscr{F}_{q}\{f\}(\boldsymbol{\omega})\right|^{2} \omega_{2}^{2} d \boldsymbol{\omega} \\
& \geq(2 \pi)^{2} \\
&\left.\cdot\left|\frac{1}{2} \int_{\mathbb{R}^{2}}\right| f(\mathbf{x})\right|^{2} d \mathbf{x}-\left.\int_{\mathbb{R}^{2}}\left|x_{1} x_{2} \frac{\overline{\partial f(\mathbf{x})}}{\partial x_{2}} \frac{\partial f(\mathbf{x})}{\partial x_{1}}\right| d \mathbf{x}\right|^{2} .
\end{aligned}
$$

It is shown that the quaternionic Gabor filters minimize the above uncertainty. Analogously, we get the uncertainty principle associated with the right-sided QFT in the following lemma.

Lemma 8 (the right-sided QFT uncertainty principle). Under the hypotheses of Theorem 7, one has the following inequality:

$$
\begin{aligned}
& \int_{\mathbb{R}^{2}} x_{1}^{2}|f(\mathbf{x})|^{2} x_{2}^{2} d \mathbf{x} \int_{\mathbb{R}^{2}} \omega_{1}^{2} \mid \mathscr{F}_{q}^{r}\left\{f_{0}+\mathbf{i} f_{1}\right\}(\boldsymbol{\omega}) \\
& +\left.\mathscr{F}_{q}^{r}\left\{\mathbf{j} f_{2}+\mathbf{k} f_{3}\right\}\left(-\omega_{1}, \omega_{2}\right)\right|^{2} \omega_{2}^{2} d \boldsymbol{\omega} \geq(2 \pi)^{2} \mid \frac{1}{2} \\
& \cdot \int_{\mathbb{R}^{2}}|f(\mathbf{x})|^{2} d \mathbf{x}-\left.\int_{\mathbb{R}^{2}}\left|x_{1} x_{2} \frac{\overline{\partial f(\mathbf{x})}}{\partial x_{2}} \frac{\partial f(\mathbf{x})}{\partial x_{1}}\right| d \mathbf{x}\right|^{2} .
\end{aligned}
$$

Theorem 7 has been recently generalized in the context of the QLCT by the authors of [30]. Our generalization is given by the following theorem.

Theorem 9 (the two-sided QLCT uncertainty principle). Under the assumptions of Theorem 7, one has

$$
\begin{aligned}
& \int_{\mathbb{R}^{2}} x_{1}^{2}|f(\mathbf{x})|^{2} x_{2}^{2} d \mathbf{x} \int_{\mathbb{R}^{2}} \omega_{1}^{2}\left|L_{A_{1}, A_{2}}^{\boxplus}\{f\}(\boldsymbol{\omega})\right|^{2} \omega_{2}^{2} d \boldsymbol{\omega} \\
& \geq\left.\left|b_{1} b_{2}\right|^{2}\left|\frac{1}{2} \int_{\mathbb{R}^{2}}\right| f(\mathbf{x})\right|^{2} d \mathbf{x} \\
& -\left.\int_{\mathbb{R}^{2}}\left|x_{1} x_{2} \frac{\partial \tilde{f}(\mathbf{x})}{\partial x_{2}} \frac{\partial \tilde{f}(\mathbf{x})}{\partial x_{1}}\right| d \mathbf{x}\right|^{2},
\end{aligned}
$$

where $\widetilde{f}(\mathbf{x})$ is defined by

$$
\tilde{f}(\mathbf{x})=e^{\mathbf{i}\left(a_{1} / 2 b_{1}\right) x_{1}^{2}} f(\mathbf{x}) e^{\mathbf{j}\left(a_{2} / 2 b_{2}\right) x_{2}^{2}} .
$$


It is worth noting here that if $\int_{\mathbb{R}^{2}} \mid x_{1} x_{2} \overline{\partial \widetilde{f}(\mathbf{x}) / \partial x_{2}}\left(\partial \tilde{f}(\mathbf{x}) / \partial x_{1}\right)$ $\mid d \mathbf{x}=0$, (41) can be reduced to

$$
\begin{gathered}
\int_{\mathbb{R}^{2}} x_{1}^{2}|f(\mathbf{x})|^{2} x_{2}^{2} d \mathbf{x} \int_{\mathbb{R}^{2}} \omega_{1}^{2}\left|L_{A_{1}, A_{2}}^{\mathbb{Q}}\{f\}(\boldsymbol{\omega})\right|^{2} \omega_{2}^{2} d \boldsymbol{\omega} \\
\geq \frac{\left|b_{1} b_{2}\right|^{2}}{4}\left(\int_{\mathbb{R}^{2}}|f(\mathbf{x})|^{2} d \mathbf{x}\right)^{2} .
\end{gathered}
$$

Proof. By replacing $f$ by $h$ defined by (34) on both sides of (39), we immediately get

$$
\begin{aligned}
& \int_{\mathbb{R}^{2}} x_{1}^{2}|h(\mathbf{x})|^{2} x_{2}^{2} d \mathbf{x} \int_{\mathbb{R}^{2}} \omega_{1}^{2}\left|\mathscr{F}_{q}\{h\}(\boldsymbol{\omega})\right|^{2} \omega_{2}^{2} d \boldsymbol{\omega} \\
& \quad \geq(2 \pi)^{2}
\end{aligned}
$$

$$
\left.\cdot\left|\frac{1}{2} \int_{\mathbb{R}^{2}}\right| h(\mathbf{x})\right|^{2} d \mathbf{x}-\left.\int_{\mathbb{R}^{2}}\left|x_{1} x_{2} \frac{\overline{\partial h(\mathbf{x})}}{\partial x_{2}} \frac{\partial h(\mathbf{x})}{\partial x_{1}}\right| d \mathbf{x}\right|^{2} .
$$

Let $\boldsymbol{\omega}=\boldsymbol{\omega} / \mathbf{b}$; then, (44) becomes

$$
\begin{aligned}
\int_{\mathbb{R}^{2}} & x_{1}^{2}|h(\mathbf{x})|^{2} x_{2}^{2} d \mathbf{x} \int_{\mathbb{R}^{2}} \frac{\omega_{1}^{2}}{b_{1}^{2}}\left|\mathscr{F}_{q}\{h\}\left(\frac{\boldsymbol{\omega}}{\mathbf{b}}\right)\right|^{2} \frac{\omega_{2}^{2}}{b_{2}^{2}} d \frac{\boldsymbol{\omega}}{\mathbf{b}} \\
\geq & (2 \pi)^{2} \\
& \left.\cdot\left|\frac{1}{2} \int_{\mathbb{R}^{2}}\right| h(\mathbf{x})\right|^{2} d \mathbf{x}-\left.\int_{\mathbb{R}^{2}}\left|x_{1} x_{2} \frac{\overline{\partial h(\mathbf{x})}}{\partial x_{2}} \frac{\partial h(\mathbf{x})}{\partial x_{1}}\right| d \mathbf{x}\right|^{2} .
\end{aligned}
$$

In view of (34), we obtain

$$
\begin{aligned}
& \int_{\mathbb{R}^{2}} \frac{x_{1}^{2}}{(2 \pi)^{2}\left|b_{1} b_{2}\right|^{2}}|f(\mathbf{x})|^{2} x_{2}^{2} d \mathbf{x} \int_{\mathbb{R}^{2}} \frac{\omega_{1}^{2}}{b_{1}^{2}}\left|\mathscr{F}_{q}\{h\}\left(\frac{\boldsymbol{\omega}}{\mathbf{b}}\right)\right|^{2} \frac{\omega_{2}^{2}}{b_{2}^{2}} d \boldsymbol{\omega} \\
& \quad \geq\left.\frac{1}{\left|(2 \pi)^{2} b_{1} b_{2}\right|^{2}}\left|\frac{1}{2} \int_{\mathbb{R}^{2}}\right| f(\mathbf{x})\right|^{2} d \mathbf{x}-\left.\int_{\mathbb{R}^{2}}\left|x_{1} x_{2} \frac{\partial\left(e^{\mathbf{i}\left(a_{1} / 2 b_{1}\right) x_{1}^{2}} f(\mathbf{x}) e^{\left.\mathbf{j}\left(a_{2} / 2 b_{2}\right) x_{2}^{2}\right)}\right.}{\partial x_{2}} \frac{\partial\left(e^{\mathbf{i}\left(a_{1} / 2 b_{1}\right) x_{1}^{2}} f(\mathbf{x}) e^{\left.\mathbf{j}\left(a_{2} / 2 b_{2}\right) x_{2}^{2}\right)}\right.}{\partial x_{1}}\right| d \mathbf{x}\right|^{2} .
\end{aligned}
$$

This leads to

$$
\begin{aligned}
& \int_{\mathbb{R}^{2}} \frac{x_{1}^{2}}{\left|b_{1} b_{2}\right|^{4}}|f(\mathbf{x})|^{2} x_{2}^{2} d \mathbf{x} \int_{\mathbb{R}^{2}} \omega_{1}^{2}\left|\mathscr{F}_{q}\{h\}\left(\frac{\boldsymbol{\omega}}{\mathbf{b}}\right)\right|^{2} \omega_{2}^{2} d \boldsymbol{\omega} \\
& \geq\left.\frac{1}{\left|b_{1} b_{2}\right|^{2}}\left|\frac{1}{2} \int_{\mathbb{R}^{2}}\right| f(\mathbf{x})\right|^{2} d \mathbf{x}-\left.\int_{\mathbb{R}^{2}}\left|x_{1} x_{2} \frac{\partial\left(e^{\mathbf{i}\left(a_{1} / 2 b_{1}\right) x_{1}^{2}} f(\mathbf{x}) e^{\mathbf{j}\left(a_{2} / 2 b_{2}\right) x_{2}^{2}}\right)}{\partial x_{2}} \frac{\partial\left(e^{\mathbf{i}\left(a_{1} / 2 b_{1}\right) x_{1}^{2}} f(\mathbf{x}) e^{\mathbf{j}\left(a_{2} / 2 b_{2}\right) x_{2}^{2}}\right)}{\partial x_{1}}\right| d \mathbf{x}\right|^{2}
\end{aligned}
$$

Inserting (35) into (47), we easily obtain

$$
\begin{aligned}
& \int_{\mathbb{R}^{2}} \frac{x_{1}^{2}}{\left|b_{1} b_{2}\right|^{4}}|f(\mathbf{x})|^{2} x_{2}^{2} d \mathbf{x} \int_{\mathbb{R}^{2}} \omega_{1}^{2}\left|e^{-\mathbf{i}\left(d_{1} / 2 b_{1}\right) \omega_{1}^{2}} L_{A_{1}, A_{2}}^{\mathbb{M}}\{f\}(\boldsymbol{\omega}) e^{-\mathbf{j}\left(d_{2} / 2 b_{2}\right) \omega_{2}^{2}}\right|^{2} \omega_{2}^{2} d \boldsymbol{\omega} \\
& \geq\left.\frac{1}{\left|b_{1} b_{2}\right|^{2}}\left|\frac{1}{2} \int_{\mathbb{R}^{2}}\right| f(\mathbf{x})\right|^{2} d \mathbf{x}-\left.\int_{\mathbb{R}^{2}}\left|x_{1} x_{2} \frac{\overline{\partial\left(e^{\mathbf{i}\left(a_{1} / 2 b_{1}\right) x_{1}^{2}} f(\mathbf{x}) e^{\mathbf{j}\left(a_{2} / 2 b_{2}\right) x_{2}^{2}}\right)}}{\partial x_{2}} \frac{\partial\left(e^{\mathbf{i}\left(a_{1} / 2 b_{1}\right) x_{1}^{2}} f(\mathbf{x}) e^{\mathbf{j}\left(a_{2} / 2 b_{2}\right) x_{2}^{2}}\right)}{\partial x_{1}}\right| d \mathbf{x}\right|^{2}
\end{aligned}
$$

The above identity can be further simplified to

$$
\begin{aligned}
& \int_{\mathbb{R}^{2}} x_{1}^{2}|f(\mathbf{x})|^{2} x_{2}^{2} d \mathbf{x} \int_{\mathbb{R}^{2}} \omega_{1}^{2}\left|L_{A_{1}, A_{2}}^{\boxplus}\{f\}(\boldsymbol{\omega})\right|^{2} \omega_{2}^{2} d \boldsymbol{\omega} \\
& \geq\left.\left|b_{1} b_{2}\right|^{2}\left|\frac{1}{2} \int_{\mathbb{R}^{2}}\right| f(\mathbf{x})\right|^{2} d \mathbf{x}
\end{aligned}
$$

$$
-\left.\int_{\mathbb{R}^{2}}\left|x_{1} x_{2} \frac{\overline{\partial \tilde{f}(\mathbf{x})}}{\partial x_{2}} \frac{\partial \tilde{f}(\mathbf{x})}{\partial x_{1}}\right| d \mathbf{x}\right|^{2}
$$

where $\tilde{f}(\mathbf{x})$ is defined in (42). Therefore, the proof is complete. 
The above theorem is also valid for the right-sided QLCT as described in the following lemma.

Lemma 10 (the right-sided QLCT uncertainty principle). Under the assumptions of Theorem 7, one has

$$
\begin{gathered}
\int_{\mathbb{R}^{2}} x_{1}^{2}|f(\mathbf{x})|^{2} x_{2}^{2} d \mathbf{x} \int_{\mathbb{R}^{2}} \omega_{1}^{2} \mid L_{A_{1}, A_{2}}^{(r), \mathbf{H}}\left\{f_{0}+\mathbf{i} f_{1}\right\}(\boldsymbol{\omega}) \\
+\left.L_{A_{1}^{*}, A_{2}^{*}}^{(r), \mathbf{H}}\left\{\mathbf{j} f_{2}+\mathbf{k} f_{3}\right\}\left(-\omega_{1}, \omega_{2}\right)\right|^{2} \omega_{2}^{2} d \boldsymbol{\omega} \geq\left|b_{1} b_{2}\right|^{2} \mid \frac{1}{2} \\
\cdot \int_{\mathbb{R}^{2}}|f(\mathbf{x})|^{2} d \mathbf{x}-\left.\int_{\mathbb{R}^{2}}\left|x_{1} x_{2} \frac{\overline{\partial \tilde{f}(\mathbf{x})}}{\partial x_{2}} \frac{\partial \tilde{f}(\mathbf{x})}{\partial x_{1}}\right| d \mathbf{x}\right|^{2}
\end{gathered}
$$

Proof. The proof follows directly from (37).

Now, we define a module of $\mathscr{F}_{q}\{f\}(\boldsymbol{\omega})$ as

$$
\begin{aligned}
& \left|\mathscr{F}_{q}\{f\}(\boldsymbol{\omega})\right|_{q}=\left(\left|\mathscr{F}_{q}\left\{f_{0}\right\}(\boldsymbol{\omega})\right|^{2}+\left|\mathscr{F}_{q}\left\{f_{1}\right\}(\boldsymbol{\omega})\right|^{2}\right. \\
& \left.+\left|\mathscr{F}_{q}\left\{f_{2}\right\}(\boldsymbol{\omega})\right|^{2}+\left|\mathscr{F}_{q}\left\{f_{3}\right\}(\boldsymbol{\omega})\right|^{2}\right)^{1 / 2},
\end{aligned}
$$

where

$$
\mathscr{F}_{q}\left\{f_{i}\right\}(\boldsymbol{\omega})=\int_{\mathbb{R}^{2}} e^{-\mathbf{i} \omega_{1} x_{1}} f_{i}(\mathbf{x}) e^{-\mathbf{j} \omega_{2} x_{2}} d \mathbf{x},
$$

$$
i=0,1,2,3 \text {. }
$$

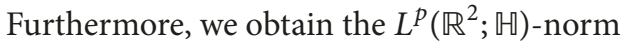

$$
\left\|\mathscr{F}_{q}\{f\}\right\|_{q, p}=\left(\int_{\mathbb{R}^{2}}\left|\mathscr{F}_{q}\{f\}(\boldsymbol{\omega})\right|_{q}^{p} d \mathbf{x}\right)^{1 / p} .
$$

It should be noticed that if $\mathscr{F}_{q}\left\{f_{i}\right\}, i=0,2,3$, is realvalued function, then (53) reduces to

$$
\left\|\mathscr{F}_{q}\{f\}\right\|_{q, p}=\left\|\mathscr{F}_{q}\{f\}\right\|_{p},
$$

where

$$
\left\|\mathscr{F}_{q}\{f\}\right\|_{p}=\left(\int_{\mathbb{R}^{2}}\left|\mathscr{F}_{q}\{f\}(\boldsymbol{\omega})\right|^{p} d \mathbf{x}\right)^{1 / p} .
$$

By Riesz's interpolation theorem, one can get the Hausdorff-Young inequality related to the QFT (see [10]):

$$
\left\|\mathscr{F}_{q}\{f\}\right\|_{q, p^{\prime}} \leq\|f\|_{p}
$$

holds for $1 \leq p \leq 2$ with $1 / p+1 / p^{\prime}$. This gives the following important theorem.

Theorem 11 (Hausdorff-Young inequality). If $1 \leq p \leq 2$ and letting $p^{\prime}$ be such that $1 / p+1 / p^{\prime}=1$, then, for all $f \in L^{p}\left(\mathbb{R}^{2} ; \mathbb{\boxplus}\right)$, it holds that

$$
\left\|L_{A_{1}, A_{2}}^{\mathbb{U}}\{f\}\right\|_{q, p^{\prime}} \leq \frac{\left|b_{1} b_{2}\right|^{-1 / 2+1 / p^{\prime}}}{2 \pi}\|f\|_{p},
$$

where

$$
\left\|L_{A_{1}, A_{2}}^{\boxplus}\{f\}\right\|_{q, p^{\prime}}=\left(\int_{\mathbb{R}^{2}}\left|L_{A_{1}, A_{2}}^{\boxplus}\{f\}(\boldsymbol{\omega})\right|_{q}^{p^{\prime}} d \boldsymbol{\omega}\right)^{1 / p^{\prime}} .
$$

Proof. From the Hausdorff-Young inequality for the QFT, we have

$$
\left(\int_{\mathbb{R}^{2}}\left|\mathscr{F}_{q}\{f\}(\boldsymbol{\omega})\right|_{q}^{p^{\prime}} d \boldsymbol{\omega}\right)^{1 / p^{\prime}} \leq\left(\int_{\mathbb{R}^{2}}|f(\mathbf{x})|^{p} d \mathbf{x}\right)^{1 / p} .
$$

Based on the arguments used in the proof of Theorem 9, we immediately get

$$
\begin{aligned}
& \left(\int_{\mathbb{R}^{2}}\left|\mathscr{F}_{q}\{h\}(\boldsymbol{\omega})\right|_{q}^{p^{\prime}} d \boldsymbol{\omega}\right)^{1 / p^{\prime}} \leq\left(\int_{\mathbb{R}^{2}}|h(\mathbf{x})|^{p} d \mathbf{x}\right)^{1 / p} \\
& \cdot\left(\int_{\mathbb{R}^{2}} \frac{1}{\left|b_{1} b_{2}\right|}\left|\mathscr{F}_{q}\{h\}\left(\frac{\boldsymbol{\omega}}{\mathbf{b}}\right)\right|_{q}^{p^{\prime}} d \boldsymbol{\omega}\right)^{1 / p^{\prime}} \\
& \leq\left(\int_{\mathbb{R}^{2}} \mid \frac{e^{-\mathbf{i}(\pi / 4)}}{\sqrt{2 \pi b_{1}}} e^{\left(\mathbf{i} a_{1} / 2 b_{1}\right) x_{1}^{2}} f(\mathbf{x}) \frac{e^{-\mathbf{j}(\pi / 4)}}{\sqrt{2 \pi b_{2}}}\right. \\
& \left.\left.\cdot e^{\left(\mathbf{j} a_{2} / 2 b_{2}\right) x_{2}^{2}}\right|^{p} d \mathbf{x}\right)^{1 / p} \frac{1}{\left|b_{1} b_{2}\right|^{1 / p^{\prime}}}\left(\int_{\mathbb{R}^{2}} \mid \mathscr{F}_{q}\{h\}\right. \\
& \left.\left.\cdot\left(\frac{\boldsymbol{\omega}}{\mathbf{b}}\right)\right|_{q} ^{p^{\prime}} d \boldsymbol{\omega}\right)^{1 / p^{\prime}} \\
& \leq \frac{1}{2 \pi\left|b_{1} b_{2}\right|^{1 / 2}}\left(\int_{\mathbb{R}^{2}}|f(\mathbf{x})|^{p} d \mathbf{x}\right)^{1 / p} .
\end{aligned}
$$

Hence,

$$
\begin{aligned}
& \left(\int_{\mathbb{R}^{2}}\left|e^{-\left(\mathbf{i} d_{1} / 2 b_{1}\right) \omega_{1}^{2}} L_{A_{1}, A_{2}}^{\boxplus 1}\{f\}(\boldsymbol{\omega}) e^{-\left(\mathrm{j} d_{2} / 2 b_{2}\right) \omega_{2}^{2}}\right|_{q}^{p^{\prime}} d \boldsymbol{\omega}\right)^{1 / p^{\prime}} \\
& \quad \leq \frac{\left|b_{1} b_{2}\right|^{-1 / 2+1 / p^{\prime}}}{2 \pi}\left(\int_{\mathbb{R}^{2}}|f(\mathbf{x})|^{p} d \mathbf{x}\right)^{1 / p} .
\end{aligned}
$$

Or, equivalently,

$$
\begin{aligned}
& \left(\int_{\mathbb{R}^{2}}\left|L_{A_{1}, A_{2}}^{\mathbb{H}}\{f\}(\boldsymbol{\omega})\right|_{q}^{p^{\prime}} d \boldsymbol{\omega}\right)^{1 / p^{\prime}} \\
& \quad \leq \frac{\left|b_{1} b_{2}\right|^{-1 / 2+1 / p^{\prime}}}{2 \pi}\left(\int_{\mathbb{R}^{2}}|f(\mathbf{x})|^{p} d \mathbf{x}\right)^{1 / p} .
\end{aligned}
$$

Thus, the theorem is completely proved.

\section{Conclusion}

In this paper, we derived a version of the uncertainty principle for the QLCT using a relation between the QFT and the QLCT. We presented Hausdorff-Young inequality associated with the QLCT. This inequality is very useful for establishing a variation on Heisenberg's uncertainty principle related to the QLCT.

\section{Conflicts of Interest}

The authors declare that there are no conflicts of interest regarding the publication of this paper. 


\section{Acknowledgments}

This work is supported by Research Collaboration Between Universities (PEKERTI) (no. 622.48/UN28.2/PL/2017) of Indonesia.

\section{References}

[1] L. Debnath and F. A. Shah, Wavelet Transforms and Their Applications, Birkhäuser, USA, 2010.

[2] S. Mallat, A Wavelet Tour of Signal Processing, Academic Press, San Diego, Calif, USA, 2nd edition, 1998.

[3] T. G. Feeman, The Mathematics of Medical Imaging, Springer Science+Business Media, New York, NY, USA, 2010.

[4] K. Grochenig, Foundations of Time-Frequency Analysis, Birkhauser, Boston, Mass, USA, 2000.

[5] M. Bahri, R. Ashino, and R. Vaillancourt, "Continuous quaternion Fourier and wavelet transforms," International Journal of Wavelets, Multiresolution and Information Processing, vol. 12, no. 4, Article ID 1460003, 21 pages, 2014.

[6] M. Bahri, A. Lawi, N. Aris, A. F. Saleh, and M. Nur, "Relationships between convolution and correlation for fourier transform and quaternion fourier transform," International Journal of Mathematical Analysis, vol. 7, no. 41-44, pp. 2101-2109, 2013.

[7] M. Bahri, "Quaternion algebra-valued wavelet transform," Applied Mathematical Sciences, vol. 5, no. 69-72, pp. 3531-3540, 2011.

[8] M. Bahri, "Construction of quaternion-valued wavelets," Matematika (Johor Bahru), vol. 26, no. 1, pp. 107-114, 2010.

[9] H. De Bie, N. De Schepper, T. A. Ell, K. Rubrecht, and S. J. Sangwine, "Connecting spatial and frequency domains for the quaternion Fourier transform," Applied Mathematics and Computation, vol. 271, pp. 581-593, 2015.

[10] L.-P. Chen, K. I. Kou, and M.-S. Liu, "Pitt's inequality and the uncertainty principle associated with the quaternion fourier transform," Journal of Mathematical Analysis and Applications, vol. 423, no. 1, pp. 681-700, 2015.

[11] E. M. Hitzer, "Directional uncertainty principle for quaternion fourier transform," Advances in Applied Clifford Algebras (AACA), vol. 20, no. 2, pp. 271-284, 2010.

[12] E. M. Hitzer, "Quaternion Fourier transform on quaternion fields and generalizations," Advances in Applied Clifford Algebras (AACA), vol. 20, no. 3, pp. 497-517, 2007.

[13] E. Hitzer, "General two-sided quaternion fourier transform, convolution and mustard convolution," Advances in Applied Clifford Algebras (AACA), vol. 27, no. 1, pp. 381-395, 2017.

[14] M. Fei, Y. Xu, and J. Yan, "Real Paley-Wiener theorem for the quaternion Fourier transform," Complex Variables and Elliptic Equations. An International Journal, vol. 62, no. 8, pp. 10721080, 2017.

[15] Y. Yang, P. Dang, and T. Qian, “Tighter uncertainty principles based on quaternion Fourier transform," Advances in Applied Clifford Algebras (AACA), vol. 26, no. 1, pp. 479-497, 2016.

[16] T. A. Ell, "Quaternion-fourier transforms for analysis of twodimensional linear time-invariant partial differential systems," in Proceeding of the 32nd Conference on Decision and Control, IEEE Control Systems Society, pp. 1830-1841, December 1993.

[17] A. M. Grigoryan and S. S. Agaian, "Tensor transform-based quaternion fourier transform algorithm," Information Sciences, vol. 320, pp. 62-74, 2015.
[18] V. R. Dubey, "Quaternion Fourier transform for colour images," International Journal of Computer Science and Information Technologies, vol. 5, no. 3, pp. 4411-4416, 2014.

[19] S. Sangwine and T. Ell, "Hypercomplex Fourier transforms of color images," IEEE Transactions on Image Processing, vol. 16, no. 1, pp. 22-35, 2007.

[20] M. Bahri, Zulfajar, and R. Ashino, "Convolution and correlation theorem for linear canonical transform and properties," Information, vol. 17, no. 6B, pp. 2509-2521, 2014.

[21] D. Wei, Q. Ran, and Y. Li, "A convolution and correlation theorem for the linear canonical transform and its application," Circuits, Systems and Signal Processing, vol. 31, no. 1, pp. 301-312, 2012.

[22] S. Xu, L. Feng, Y. Chai, Y. Hu, and L. Huang, "The properties of generalized offset linear canonical Hilbert transform and its applications," International Journal of Wavelets, Multiresolution and Information Processing, vol. 15, no. 4, Article ID 1750031, 21 pages, 2017.

[23] M. Bahri and R. Ashino, "Logarithmic uncertainty principle for quaternion linear canonical transform," in Proceedings of the 2016 International Conference on Wavelet Analysis and Pattern Recognition, ICWAPR 2016, pp. 140-145, South Korea, July 2016.

[24] K. I. Kou, J.-Y. Ou, and J. Morais, "On uncertainty principle for quaternionic linear canonical transform," Abstract and Applied Analysis, vol. 2013, Article ID 725952, 14 pages, 2013.

[25] X.-X. Hu and K. I. Kou, "Quaternion Fourier and linear canonical inversion theorems," Mathematical Methods in the Applied Sciences, vol. 40, no. 7, pp. 2421-2440, 2017.

[26] M. Bahri and R. Ashino, "A simplified proof of uncertainty principle for quaternion linear canonical transform," Abstract and Applied Analysis, Article ID 5874930, Art. ID 5874930, 11 pages, 2016.

[27] K. I. Kou, J. Ou, and J. Morais, "Uncertainty principles associated with quaternionic linear canonical transforms," Mathematical Methods in the Applied Sciences, vol. 39, no. 10, pp. 27222736, 2016.

[28] T. Bülow, Hypercomplex Spectral Signal Representations for the Processing and Analysis of Images [PhD Thesis], University of Kiel, Germany, 1999.

[29] M. Bahri, "A modified uncertainty principle for two-sided quaternion Fourier transform," Advances in Applied Clifford Algebras (AACA), vol. 26, no. 2, pp. 513-527, 2016.

[30] Y.-N. Zhang and B.-Z. Li, "Novel uncertainty principles for two-sided quaternion linear canonical transform," Advances in Applied Clifford Algebras (AACA), vol. 28, no. 15, 2018. 


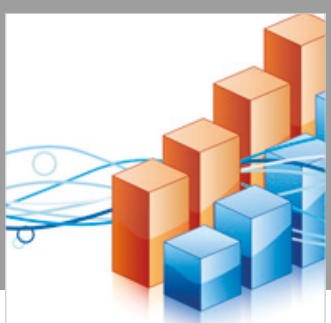

Advances in

Operations Research

\section{-n-m}
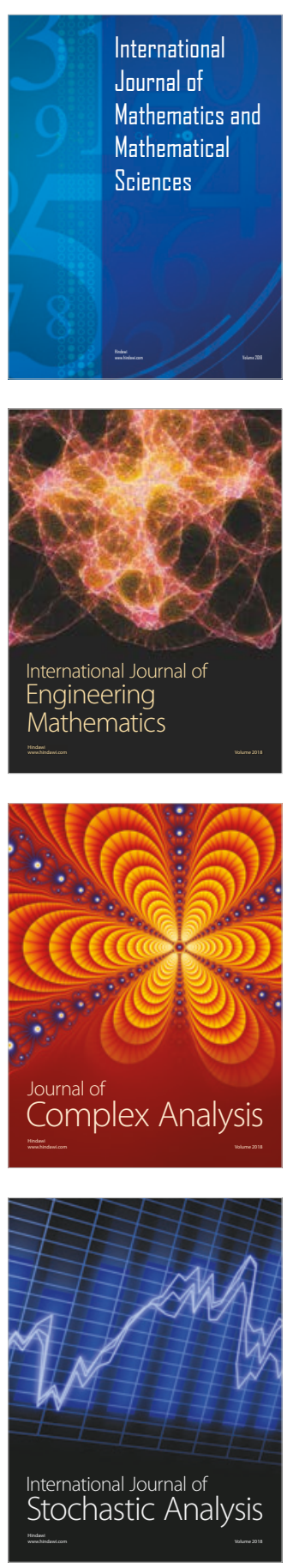
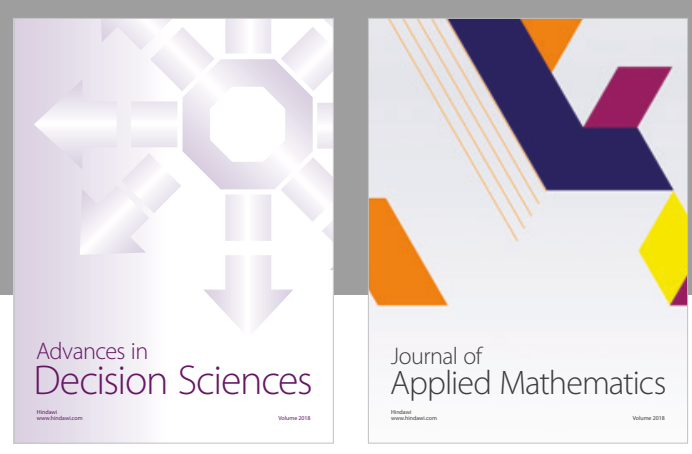

Journal of

Applied Mathematics
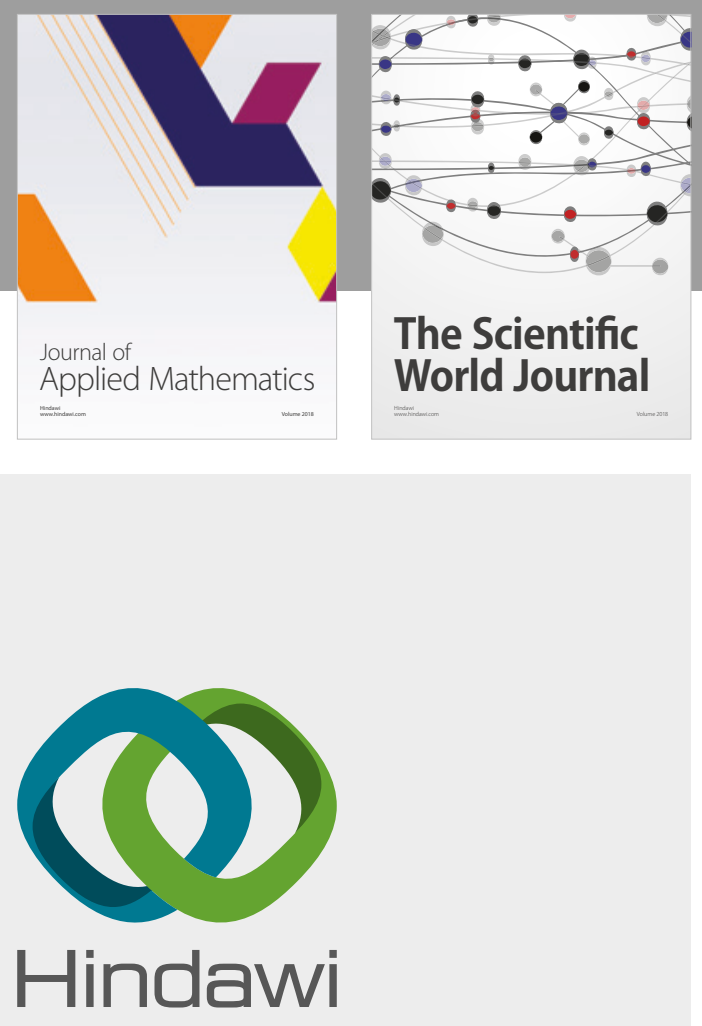

Submit your manuscripts at

www.hindawi.com

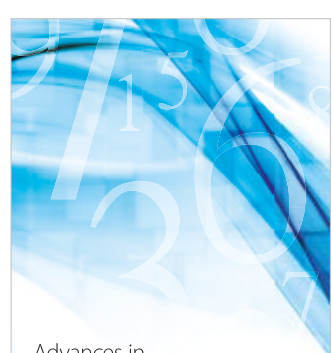

Advances in
Numerical Analysis
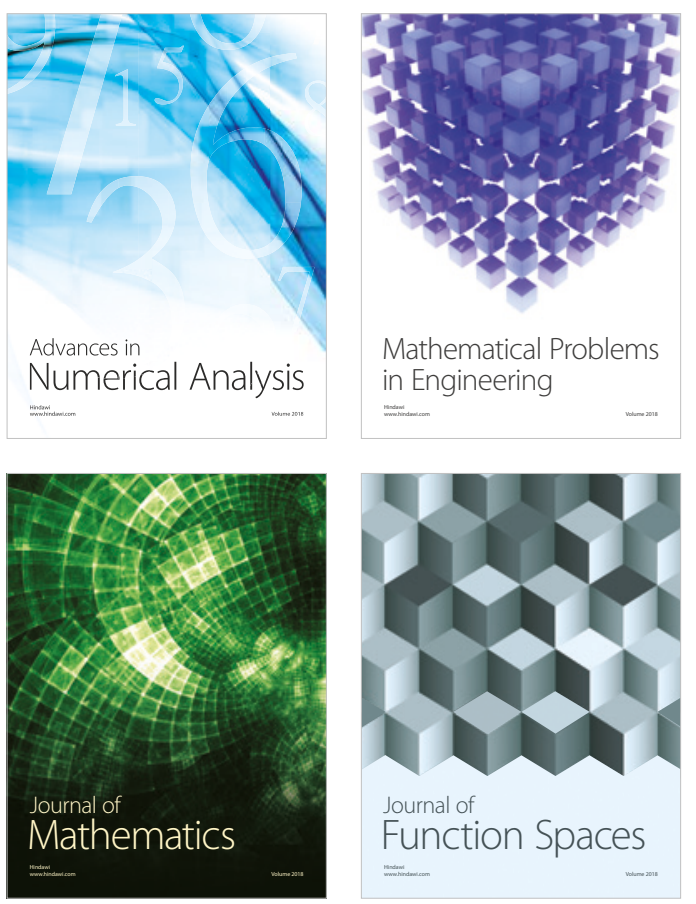

Mathematical Problems in Engineering

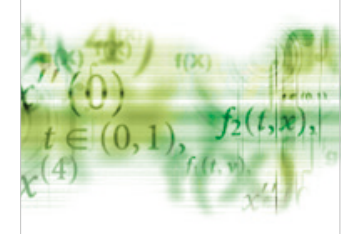

International Journal of

Differential Equations

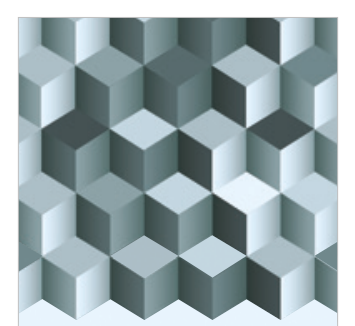

Journal of

Function Spaces

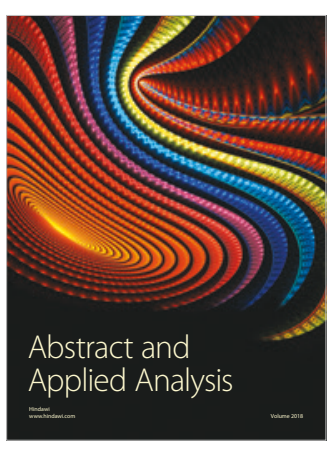

The Scientific

World Journal

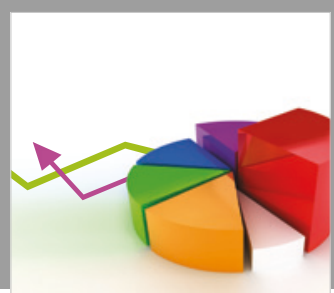

Journal of

Probability and Statistics
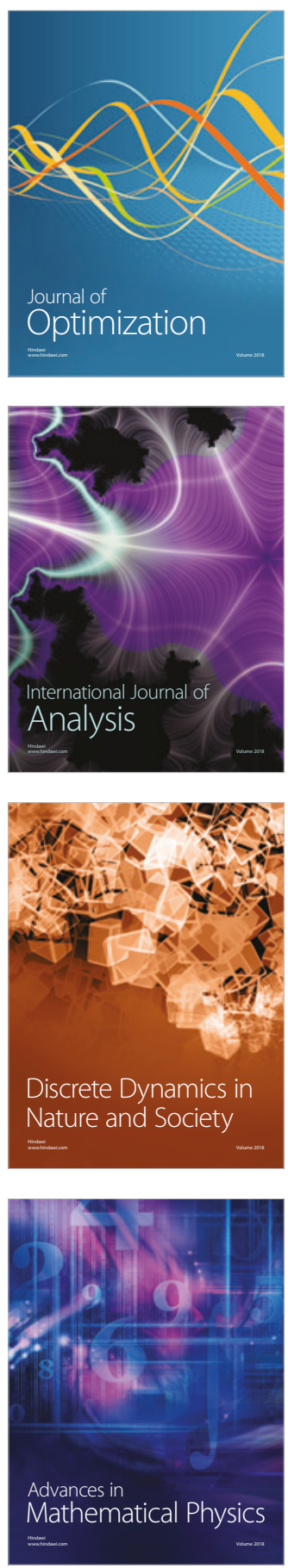